\title{
O Psicólogo na Atenção Básica: Uma Incursão Pelas Políticas Públicas de Saúde Brasileiras ${ }^{1}$
}

The Psychologist in Primary Care: An Incursion in the Brazilian Health Public Policy

El Psicólogo en la Atención Básica: Una Incursión por las Políticas Públicas de Salud Brasileñas

Elisangela Böing

Prefeitura Municipal

de Florianópolis

Maria Aparecida Crepaldi

Universidade Federal de Santa Catarina

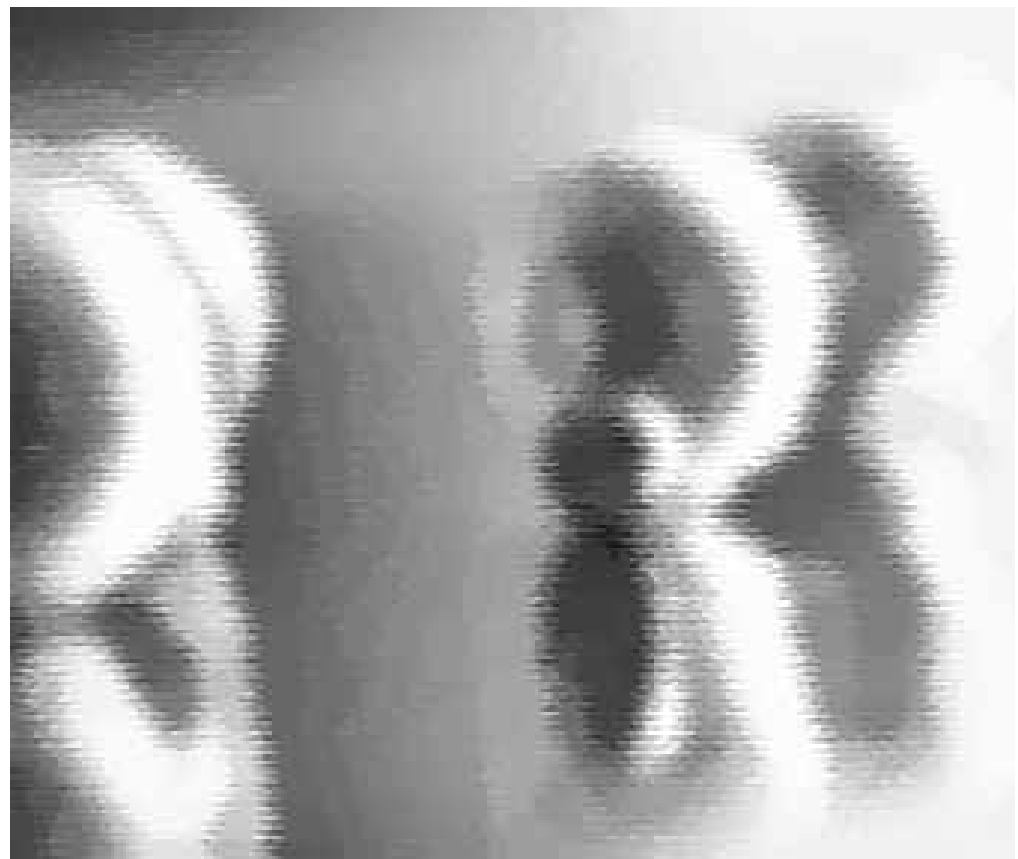


Resumo: Este artigo relata uma pesquisa cujo objetivo foi identificar, na legislação federal de saúde, em que medida e de que forma as políticas públicas contemplam a atuação do psicólogo na atenção básica no Brasil, ampliando a compreensão da inserção dos psicólogos no Sistema Único de Saúde (SUS). Utilizouse o método de pesquisa documental, e os resultados mostraram que as políticas tratam da inclusão do psicólogo nas equipes de saúde em apenas 14 dos 964 documentos pesquisados. Há prevalência de inclusão nos níveis secundário e terciário de atenção. No modelo de atenção preconizado, a relação que o profissional de Psicologia estabelece com a atenção básica se dá através do Apoio Matricial às equipes de saúde da família. Entretanto, identificou-se que este constitui atuação de nível secundário. Concluiu-se que a configuração das políticas de saúde não favorece a efetivação de uma atuação do psicólogo condizente com as demandas da atenção básica. Entende-se que o SUS deveria contar com psicólogos nas unidades locais de saúde, inseridos nas equipes de saúde da família que desenvolvessem trabalho interdisciplinar voltado para a atenção integral, e com psicólogos especialistas locados nos núcleos e nos centros nos níveis secundário e terciário.

Palavras-chave: Atuação do psicólogo. Serviços de saúde. Políticas públicas. Atenção primária á saúde. SUS.

\begin{abstract}
This article reports a study whose aim was to identify in federal health legislation to what extent and how the public health policies include the psychologist in primary care in Brazil in order to increase the understanding of the psychologists' integration in the Unified Health System. The method of documentary research was used and the results showed that the policies mention the psychologist's inclusion in the health care teams in only 14 out of the 964 documents searched. There's a prevalence of their inclusion in the secondary and tertiary health care levels. In the model of care recommended, the relationship that the psychologist establishes with the primary care is given by the matricial support for family health teams. However, it was identified that the matricial support model is a secondary level practice. Thus, it was concluded that the configuration of public health policies don't conduct to the effective psychologist's performance that the primary care demands. It is understood that the public health care system should have psychologists in local health units, inserted in the health care family teams, developing an interdisciplinary work focused on integral health attention and psychologists in the nuclei and centers of secondary and tertiary health care levels.
\end{abstract}

Keywords: Psychologist performance. Helth services. Public policies. Primary health care. SUS.

Resumen: Este artículo relata una pesquisa cuyo objetivo fue identificar, en la legislación federal de salud, en qué medida y de qué forma las políticas públicas contemplan la actuación del psicólogo en la atención básica en el Brasil, ampliando la comprensión de la inserción de los psicólogos en el Sistema Único de Salud (SUS). Se utilizó el método de pesquisa documental, y los resultados mostraron que las políticas tratan de la inclusión del psicólogo en los equipos de salud en solamente 14 de los 964 documentos pesquisados. Hay prevalencia de inclusión en los niveles secundario y terciario de atención. En el modelo de atención preconizado, la relación que el profesional de Psicología establece con la atención básica se da a través del Apoyo Matricial a los equipos de salud de la familia. Entretanto, se identificó que éste constituye actuación de nivel secundario. Se concluyó que la configuración de las políticas de salud no favorece la efectivación de una actuación del psicólogo condecente con las demandas de la atención básica. Se entiende que el SUS debería contar con psicólogos en las unidades locales de salud, inseridos en los equipos de salud de la familia que desarrollasen trabajo interdisciplinario dirigido hacia la atención integral, y con psicólogos especialistas destinados en los núcleos y en los centros en los niveles secundario y terciario.

Palabras clave: Actuación del psicólogo. Servicios de salud. Políticas públicas. Atención primaria de salud. SUS.

No cenário atual, as políticas públicas de saúde brasileiras são organizadas e regidas pelas leis do Sistema Único de Saúde (SUS). O SUS está amparado em uma vasta legislação, cujo tripé principal é formado pela Constituição Federal de 1988 e pelas Leis no 8.080 e no 8.142, ambas de 1990; complementarmente, existiram várias normas operacionais. As portarias ministeriais são importantes instrumentos de regulação de políticas, e visam a definir instruções para a execução das leis aprovadas pelo Poder Legislativo, tal como previsto na Constituição Federal de 1988. No contexto do setor saúde, as portarias têm assumido um papel de grande destaque, não só pelo quantitativo de documentos apresentados a partir dos últimos anos da década de 90 mas principalmente 
pelo forte poder de indução que esse instrumento assumiu na definição da política setorial (Baptista, 2007).

Os principais artigos das leis no 8080 e $\mathrm{n}$ 8142 estabelecem um conceito ampliado de saúde, incorporando fatores do meio físico, socioeconômico e cultural e oportunidades de acesso aos serviços de promoção, proteção e recuperação da saúde. Essas leis legitimaram o direito de todos, sem qualquer discriminação, às ações de saúde, cabendo ao governo garantir esse direito, e estabeleceram os princípios do SUS: universalidade; acessibilidade e coordenação do cuidado; vínculo e continuidade; integralidade da atenção; responsabilização; humanização; equidade e participação social. No contexto da atenção básica, vem se concretizando a Estratégia de Saúde da Família (ESF), proposta em 1994, como um modelo de atenção substitutivo ao modelo biomédico, tradicional, indivíduo-centrado (Andrade, Soares, \& Cordoni Jr., 2001; Brasil, 1990, 1998, 2006a).

Cabe esclarecer o uso dos termos atenção básica e atenção primária. O primeiro tem um sentido mais amplo, e compreende ações integrais que abrangem a promoção e a proteção da saúde, a prevenção de agravos, o diagnóstico, o tratamento, a reabilitação e a manutenção da saúde. Essas ações correspondem à prática de vigilância da saúde do novo modelo de atenção preconizado pela legislação do SUS, cuja efetivação é buscada através da Estratégia de Saúde da Família (Brasil, 2006a). Sendo assim, o termo atenção básica é empregado particularmente no contexto da saúde pública do Brasil. Já o termo atenção primária é internacionalmente utilizado e tem significado mais restrito, relacionado à saúde coletiva em ações de promoção e prevenção. Neste artigo, os termos serão utilizados como sinônimos, considerando que será abordada apenas a saúde coletiva brasileira e que, nesta, o primeiro nível de atenção compreende ações integrais e tem a função de organizar todo o sistema de saúde.

Para desenvolver a atenção integral à saúde que abranja a complexidade do processo saúde-doença, o trabalho interdisciplinar se torna uma real necessidade do profissional de saúde. O conhecimento e a prática interdisciplinares surgem como alternativas de se promover a inter-relação entre as diferentes áreas de conhecimento, entre os profissionais e entre eles e o senso comum, relacionandose ao pensamento divergente que requer criatividade e flexibilidade - princípio da máxima exploração das potencialidades de cada ciência e da compreensão de seus limites. A saúde deve ser vista como ponto de partida e de chegada para a intervenção profissional. O saber interdisciplinar propicia ao profissional uma visão que transcenda a especificidade do seu saber, e sua atuação se torna ampla e contextualizada, possibilitando ao mesmo a compreensão das implicações sociais de sua prática para que esta possa se tornar realmente um produto coletivo e eficaz (Gomes, 1997).

Através do princípio da integralidade, portanto, o SUS abre portas para novos atores nas equipes de saúde. Para cuidar da saúde de forma integral, torna-se imprescindível que, no primeiro nível de atenção, haja equipes interdisciplinares que desenvolvam ações intersetoriais. O psicólogo, nesse contexto, oferece uma importante contribuição na compreensão contextualizada e integral do indivíduo, das famílias e da comunidade.

Desde 1990, com a Declaração de Caracas, enfatiza-se a reestruturação da atenção psiquiátrica vinculada à atenção primária à saúde e na constituição de redes de apoio social e serviços comunitários que possam dar suporte aos indivíduos em seus contextos de vida. Inserido nesse contexto, a Estratégia 
de Saúde da Família configura campo de práticas e de produção de novos modos de cuidado em saúde mental, na medida em que tem como proposta a produção de cuidados dentro dos princípios da integralidade, da interdisciplinaridade, da intersetorialidade e da territorialidade. A articulação entre saúde mental e atenção básica é um desafio a ser enfrentado atualmente, pois a melhoria da assistência prestada e a ampliação do acesso da população aos serviços com garantia de continuidade da atenção dependem da efetivação dessa articulação (Coimbra, Oliveira, Vila, \& Almeida, 2005; Dimenstein, Santos, Brito, Severo, \& Morais, 2005; Ferrioli, Marturano, \& Puntel, 2007; Franca \& Viana, 2006; Organização Mundial de Saúde [OMS], 2001).

No contexto das políticas públicas de saúde atuais, da Estratégia de Saúde da Família e da atuação de equipes interdisciplinares na atenção básica surgiu o tema da pesquisa descrita neste artigo: a atuação do psicólogo na Atenção Básica à Saúde (Böing, 2009).

Estudos sobre a caracterização da atuação do psicólogo, no contexto da atenção primária no Brasil, mostram, de forma geral, uma atuação que não atende as demandas da saúde coletiva em função da transposição do modelo clínico tradicional sem a necessária contextualização que esse cenário requer. Sendo assim, os profissionais de Psicologia enfrentam o grande desafio de redimensionamento de suas práticas. A necessidade é de complementação e de superação da formação acadêmica no sentido de uma efetiva flexibilização das tecnologias para o desenvolvimento de práticas psicológicas condizentes com esse contexto de atuação a fim de se lidar com uma realidade desafiadora e complexa. $\mathrm{Na}$ redefinição de suas práticas, o psicólogo deve ser capaz de contribuir para a formulação e a implantação de novas políticas (Dimenstein,
1998, 2000, 2001, 2003; Franco \& Mota, 2003; Lima, 2005; Moré, 2000; Moré \& Macedo, 2006; Oliveira et al., 2005; Trindade e Teixeira, 2000b).

Durante muito tempo, os cursos de Psicologia formaram profissionais apolíticos, e ressalta-se a necessidade da formação de profissionais críticos, e não somente técnicos. De fato, quantos cursos de Psicologia têm a questão das políticas públicas como eixo articulador? Quantos têm ao menos disciplinas que abordam as reformas, sanitária e psiquiátrica? Sem tais discussões no processo de formação, os psicólogos tendem a não se ver como parte desse processo. O desenvolvimento de uma posição ética e política é uma das características necessárias para a sustentação do projeto do SUS e de uma atuação consistente na Estratégia de Saúde da Família (Benevides, 2005; Dimenstein, 1998, 2000, 2003; Lima, 2005; Oliveira, et al., 2005; Romagnoli, 2006).

Para os psicólogos, pela sua relativa recente inserção no setor saúde - década de 90 ainda não há uma definição clara do seu papel em cada um dos níveis de atenção, o que resulta em desconhecimento das possibilidades de atuação. Essencialmente, a atuação na atenção básica se caracteriza pelo desenvolvimento de um trabalho da equipe de saúde na e com a comunidade através do modelo da vigilância da saúde, focando, sobretudo, ações de promoção à saúde e trabalhando também com prevenção e atenção curativa (Böing, Crepaldi, \& Moré, 2009).

Não há, na prática, um local definido para o psicólogo no nível primário de atenção; fazem parte da equipe mínima de saúde da família apenas o enfermeiro e o médico (clínico geral), considerados profissionais de saúde de nível superior, sendo que a presença do 
odontólogo é garantida pela determinação da existência de uma equipe de saúde bucal para cada equipe de saúde da família (Brasil, 2006a). Movimentos da categoria dos psicólogos promoveram discussões acerca do papel do profissional de Psicologia na saúde coletiva, debatendo se este deve participar das equipes matriciais de apoio às equipes de saúde da família ou ser incluído nessas equipes e atuar segundo a Estratégia de Saúde da Família, na unidade local de saúde (Psicologia: Ciência e Profissão, 2006; Conselho Regional de Psicologia - 12 $2^{a}$ Região, 2007; Romagnoli, 2006); propostas do Ministério da Saúde apontam o modelo de equipes matriciais (Brasil, 2004a, 2004b, 2004c; 2006b; 2007a; 2008). O que se evidencia nessas discussões é a falta de conhecimento, por parte dos gestores, dos demais profissionais de saúde, dos próprios psicólogos e de seus representantes, do potencial das contribuições da Psicologia no nível primário de atenção e, sobretudo, dos aspectos essenciais que caracterizam um trabalho nesse nível.

Muitos são os fatores que interagem recursivamente para a atual situação da Psicologia na saúde coletiva, indefinida e insatisfatória, sobretudo na atenção básica. Um dos fatores mais debatidos refere-se à formação dos psicólogos e dos demais profissionais de saúde. Para responder essa questão, foram estabelecidas parcerias, em várias regiões do País, entre Ministério da Saúde, universidades e prefeituras municipais (secretarias municipais de saúde) para a criação de cursos de especialização e residências em saúde da família com o objetivo de capacitar profissionais de saúde, dentre eles o psicólogo, para o desenvolvimento de um trabalho em conformidade com o modelo da ESF. Outra medida foi a ampliação do Programa Nacional de Reorientação da Formação em Saúde - Pró-Saúde (Portaria no 3.019/2007) para os demais cursos de graduação da área da saúde, além dos cursos de Medicina, enfermagem e odontologia, visando a incentivar transformações do processo de formação, de geração de conhecimentos e de prestação de serviços à comunidade para abordagem integral na atenção à saúde.

O que se percebe, na prática, é que os profissionais, mesmo após dois anos de formação e de treinamento específicos, como é o caso das residências multiprofissionais, se encontram em situação indefinida na atenção básica, pois não encontram espaço e condições para desenvolverem um trabalho condizente com o novo modelo de atenção à saúde que se pretende efetivar. Esse impasse ocorre, sobretudo, com os profissionais não tradicionalmente incluídos no setor saúde, como é o caso do psicólogo. Cabe esclarecer que o referido espaço diz respeito ao campo da atenção básica, ou seja, à unidade local de saúde e à comunidade de sua área de abrangência. E a condição primordial que falta aos psicólogos é a de fazer parte de uma equipe de saúde interdisciplinar; é a condição, portanto, de terem a possibilidade de assumir o papel de profissionais de saúde, como os demais, e não exclusivamente o de especialistas na atenção especializada.

Frente a essa situação, embora os cursos de formação e treinamento sejam estratégias fundamentais para a atuação dos profissionais de saúde de acordo com o novo modelo de atenção, não podem ser tomados como medidas únicas, pois a mudança de modelo que implica uma mudança paradigmática - é um processo complexo que envolve muitos fatores inter-relacionados.

Uma vez que a questão da formação do psicólogo tem sido bastante discutida e, de certa forma, encaminhada (cursos de especialização e residência e propostas de reformas curriculares), surge o questionamento a respeito de que outros fatores estão 
envolvidos na configuração da situação insatisfatória da Psicologia na saúde coletiva. Entende-se que a conquista de espaço e de definição de papéis para uma atuação condizente com as demandas da atenção em saúde coletiva passa, necessariamente, pelas políticas públicas, sendo, portanto, esse o foco eleito para o desenvolvimento da pesquisa com objetivo de identificar em que medida e de que forma as políticas públicas de saúde e de saúde mental contemplam a atuação do psicólogo na atenção básica à saúde no Brasil.

\section{Método}

O método de pesquisa utilizado foi a investigação e a análise documental. Esse método consiste na seleção criteriosa de documentos e de técnicas de organização, de classificação do material e de elaboração de categorias de análise, sendo todo o processo orientado pelo problema de pesquisa proposto. Através do emprego de técnicas usuais da análise de conteúdo, realizou-se um processo de codificação, de interpretação e de inferências sobre as informações contidas nos documentos e de classificação a fim de vislumbrar uma síntese (Pimentel, 2001; Valles, 1997).

\section{Procedimento de coleta de dados}

O procedimento de seleção dos documentos foi realizado em quatro etapas, sendo a última subdividida em outras duas de forma a selecionar, para análise propriamente dita, apenas os documentos que respondiam aos objetivos da pesquisa.

Primeira etapa: considerando os critérios de autenticidade e credibilidade, foram utilizados apenas documentos oficiais disponíveis no site do Ministério da Saúde/ Governo Federal (http://portal.saude.gov.br/ saude). A coleta dos documentos foi realizada no período de novembro a dezembro de
2007, sendo que todos os documentos novos publicados no site até julho de 2008 foram incluídos na pesquisa. Essa seleção inicial totalizou 964 documentos.

Segunda etapa: foram selecionados os documentos que apresentaram alguma relação com o tema da pesquisa. Para tanto, foi realizada a leitura dos documentos e, para aqueles muito extensos, a busca das seguintes palavras-chave: atenção básica, atenção primária, psicólogo, Psicologia, psicológico, psicológica, mental, mentais. Essa etapa de seleção constitui o primeiro filtro que permitiu uma redução do volume do material documental. Segundo o critério de relevância, foram selecionados todos os documentos que, pela leitura, tivessem relação com o tema de pesquisa e com todos os que apresentavam ao menos uma das palavras-chave citadas. Total: 113 documentos.

Terceira etapa: foi realizada a classificação dos documentos em categorias temáticas, considerando as partes dos documentos onde se encontravam as palavras-chave. Foram estabelecidas sete categorias, sendo, nessa etapa, selecionados apenas os documentos pertencentes à categoria diretrizes: 65 documentos.

Quarta etapa: esta se apresentou como uma etapa mista, ou seja, referente ainda à seleção dos documentos, mas, também nela, iniciouse o processo de análise. Os 65 documentos pertencentes à categoria diretrizes foram analisados com o objetivo de selecionar aqueles que apresentavam informações relevantes aos objetivos da pesquisa: os que apontavam uma caracterização do tipo de assistência exigida/planejada/preconizada pela legislação federal de saúde referente à atenção básica e à saúde mental e/ou que faziam referência ao profissional de Psicologia. Através dessa análise, foram selecionados 50 dos 65 documentos. 


\section{Análise dos dados}

Para a análise propriamente dita, utilizou-se o conjunto dos 50 documentos resultante do procedimento de seleção. Através da leitura, em uma primeira etapa de análise, foram selecionados e utilizados apenas os documentos que apresentaram informações que possibilitassem uma caracterização do modelo de atenção à saúde preconizado, com relação à atenção básica e à saúde mental: 24 documentos. Em uma segunda etapa de análise, foram selecionados documentos que sugerem, indicam ou exigem a inclusão do profissional de Psicologia na equipe de saúde: 14 documentos.

\section{Resultados}

Os resultados foram agrupados em dois temas: caracterização da assistência em saúde e inclusão do psicólogo. Neste último, foram identificados os níveis de atenção, os locais de inclusão e a atuação recomendada.

\section{Caracterização da assistência em saúde}

Equipes de saúde da família como referência e organizadores de toda a rede de atenção

A Política Nacional de Atenção Básica e os documentos que tratam do apoio matricial claramente colocam as equipes de saúde da família (nas unidades locais de saúde) como referência, ou seja, como responsáveis pela integralidade e pela continuidade do cuidado e pela organização da rede de saúde. Outros 8 documentos corroboram essa estruturação do sistema de saúde.

CAPS como referência e organizador da rede de atenção em saúde mental

Documentos específicos da legislação da saúde mental apontam os CAPS que atuam nos serviços territoriais de atenção diária em saúde mental como referência e como organizadores da rede diversificada de serviços em saúde mental, e, portanto, como reguladores da porta de entrada da rede de assistência em saúde mental de sua área, além de exercerem a função de matriciamento das equipes de atenção básica. Outros dois documentos reforçam a condição de territorialidade dos CAPS.

Nessa síntese dos resultados, é possível visualizar, de forma geral, uma incoerência entre a Política Nacional de Atenção Básica e as políticas específicas de saúde mental. A primeira define as equipes de saúde da família como referência e como organizadoras de toda a rede de atenção à saúde; já a legislação de saúde mental coloca os CAPS como portas de entrada e como organizadores dos serviços de atenção à saúde mental, evidenciando, assim, uma dicotomia entre saúde e saúde mental, como se a saúde mental formasse um sistema à parte.

\section{Inclusão do psicólogo nas equipes de saúde}

Dentre os documentos pesquisados, 14 tratam da inclusão do psicólogo nas equipes de saúde.

3.2.1. De acordo com o nível de atenção e os locais a que os documentos se referem:

A) Atenção à saúde em geral: 4 documentos tratam da saúde de forma geral e apontam a necessidade de atenção psicológica, sem especificar o nível de atenção ou o local de atuação do psicólogo.

B) Atenção básica: 2 documentos tratam da atenção básica e incluem o psicólogo na equipe mínima para atenção básica no sistema penitenciário, nos moldes da Estratégia de Saúde da Família. Ambos se referem a um mesmo local: unidades prisionais com mais de 100 detentos. 
C) Atenção secundária: 6 documentos fazem referência ao psicólogo no nível secundário de atenção e apontam 7 locais diferentes para a sua inclusão. Dentre as equipes em que o psicólogo é incluído na atenção secundária, destacam-se três: NAPS/CAPS, equipes de apoio em saúde mental e NASF, uma vez que estas desempenham o papel de apoio matricial, modelo de atenção à saúde mental na atenção básica.

Cabe esclarecer que as equipes de apoio em saúde mental e os NASF não são claramente definidos nos documentos em um nível de atenção específico. Foram classificados como atenção secundária em função de seu processo de trabalho, como explicitado abaixo (item 3.2.2).

D) Atenção terciária: Com relação ao nível terciário, 4 documentos apontam 9 locais diferentes para a inclusão do psicólogo nas equipes de saúde, dentre eles, hospitais e serviços especializados.

De forma geral, evidencia-se que as políticas públicas de saúde tratam da inclusão do profissional de Psicologia em um número reduzido de documentos - 14 dos 964 pesquisados - e nestes há uma prevalência da sua inclusão nos níveis secundário e terciário de atenção. Ressalta-se que, com relação ao primeiro nível de atenção, os documentos determinam a inclusão do psicólogo em um único local: na equipe mínima da atenção básica do sistema penitenciário.

Caracterização da atuação preconizada para o psicólogo

Com relação à atuação preconizada para o psicólogo, de acordo com o tema central da pesquisa, a análise concentrou-se na atuação na atenção básica e com relação ao modelo de apoio matricial, por ser através desse modelo de atuação que se dá a relação do profissional de Psicologia com a atenção básica.
Os dois documentos que incluem o psicólogo na equipe técnica mínima para atenção básica nas unidades penitenciárias preconizam que a atuação siga o modelo da Estratégia de Saúde da Família; compreende, portanto, ações integrais em saúde através da prática de vigilância da saúde (ações de promoção e prevenção da saúde e atenção curativa) realizada por uma equipe interdisciplinar que é responsável por uma determinada população, com a qual deve estabelecer vínculo e co-responsabilização pelo cuidado, nesse caso, uma equipe técnica, em jornada de trabalho de 20 horas semanais, para até 500 pessoas presas, em penitenciárias com mais de 100 detentos.

Seis documentos referem-se ao psicólogo como apoio matricial às equipes da atenção básica, seja nos NASF, nos CAPS ou em equipes de apoio em saúde mental (Brasil, 2004a, 2004b, 2004c; 2006b; 2007a; 2008). Ressalta-se que as equipes matriciais têm a função primordial de apoio às equipes de saúde da família, e que a abrangência das ações descritas nos documentos como responsabilidades compartilhadas requer presença do profissional matriciador no cotidiano de trabalho na comunidade, como, por exemplo, no desenvolvimento de ações conjuntas, no de ações de mobilização de recursos comunitários, na priorização de abordagens coletivas e de grupos e no trabalho do vínculo com as famílias. Essas ações se tornam inviáveis em espaços reduzidos de contato equipe matricial - equipe de saúde da família, como as reuniões semanais preconizadas no processo de trabalho de apoio matricial. Destaca-se, ainda, a discussão, com as equipes de SF, dos casos identificados que necessitam de ampliação da clínica em relação a questões subjetivas. Considerando o princípio da integralidade na atenção à saúde, não seriam todos os casos que deveriam contar com a ampliação da clínica em relação a questões subjetivas? De fato, deveriam; contudo, o processo 
de trabalho do apoio matricial impõe um limite de contato e de tempo do profissional matriciador junto à comunidade e aos profissionais de saúde da família, fazendo com que se mantenha a lógica do modelo tradicional, focado no sintoma, na doença, nos casos identificados.

Assim, o modelo de apoio matricial, apesar de se referir à atuação direta com os profissionais de saúde da família e de abranger ações características de atenção básica, representa, em função do processo de trabalho preconizado, uma atuação característica de especialidades, circunscrita, portanto, ao nível secundário de atenção. O tempo e o contato restritos, no processo de trabalho, impedem a equipe de apoio matricial de participar do cotidiano das equipes de saúde da família e da comunidade, inviabilizando a vinculação e a efetivação das ações previstas.

\section{Discussão}

Na caracterização do modelo de atenção preconizado com relação à atenção básica e à saúde mental, constatou-se, nos documentos, uma dicotomia nas políticas públicas entre saúde e saúde mental. A Política Nacional de Atenção Básica, dentre outros documentos, institui a atenção básica, através das equipes de saúde da família, como referência e organizadora de todo o sistema, enquanto documentos específicos da legislação de saúde mental colocam os CAPS como referências da rede diversificada de serviços em saúde mental, como se esta não fizesse parte do sistema de saúde em geral. Tal dicotomia pode estar relacionada a um fator histórico, como reflexo dos movimentos sanitário (que implicou a mudança de modelo de atenção à saúde) e antimanicomial, uma vez que ambos se formaram na mesma época, mas tiveram encaminhamentos paralelos, inclusive em termos de políticas públicas de saúde coletiva e de saúde mental.
Se a atenção básica, através da ESF, deve ser referência e organizadora de toda a rede de saúde, deveria, portanto, abarcar a saúde mental nas suas ações cotidianas (de promoção, prevenção e atenção curativa) e organizar o acesso aos demais níveis de atenção (CAPS, hospitais...), mantendo-se co-responsável pelo cuidado. Entende-se que atribuir ao CAPS a função de porta de entrada e de organizador dos serviços de saúde mental cria um fluxo paralelo na rede de atenção, centraliza a demanda de saúde mental nesse serviço e distancia a atenção básica das ações de saúde mental, como se a saúde mental pudesse ser separada da saúde integral. Uma das consequências diretas dessa desarticulação entre o campo de saber e as práticas da saúde mental e as ações básicas de saúde é a existência e o aumento de demanda reprimida em saúde mental, como constatado no estudo de Dimenstein et al. (2005).

Entende-se, portanto, que a atenção básica deve ser efetivamente a porta de entrada, a referência e a organizadora da rede de atenção, abarcando ações de saúde mental nas suas ações cotidianas e articulando-se com os serviços de nível secundário e terciário de atenção. Considera-se, ainda, que os CAPS III e os hospitais gerais, por se tratar de serviços de emergência em saúde mental, podem vir a cumprir também a função de porta de entrada, ou seja, uma pessoa em situação de emergência será atendida com ou sem contato prévio com a equipe da atenção básica. Atendida a crise, esta deve ser relatada para a equipe de saúde da família, que será referência e organizadora do acesso aos demais serviços da rede de atenção, mantendo-se co-responsável pelo cuidado. Vale destacar, ainda, que os documentos que discorrem sobre os CAPS trazem a diretriz da territorialidade, ou seja, instituem-nos como serviço ambulatorial de atenção diária de base comunitária que funcione segundo a lógica do território, condição essa raramente presente nesses serviços, que, na 
sua maioria, na sua tipologia, são únicos, e, portanto, referência para todo o Município a que pertencem. Essa situação traz muitos prejuízos para a concretização da rede de atenção (única), pois inviabiliza muitas ações de responsabilidade dos CAPS e dificulta a comunicação dos mesmos com a atenção básica.

Na questão específica da saúde mental na atenção básica, os documentos remetem ao modelo de apoio matricial. Ressalta-se que as ações de responsabilidade entre equipes matriciais e as equipes de saúde da família pelas quais são responsáveis constituem ações condizentes com o que se espera da atuação de um profissional na atenção básica, conforme aponta a literatura (Böing et al., 2009; Calatayud, 1991; Moré, 2005; Moré \& Macedo, 2006; Teixeira, 2000ab; Trindade, 2000; Trindade \& Teixeira, 2000a; Universidade Federal de Santa Catarina [UFSC], 2007). São ações interdisciplinares e intersetoriais que requerem participação dos profissionais matriciadores nos processos de territorialização, planejamento; acompanhamento e avaliação das atividades; exige, portanto, na prática, presença e contato constantes no cotidiano de trabalho das equipes de saúde da família e na comunidade, o que se mostra inviável considerando a abrangência da responsabilidade das equipes matriciais: uma equipe matricial para 8 a 20 equipes de saúde da família, no caso do NASF II, e uma equipe de saúde mental para 6 a 9 equipes de saúde da família, considerando, ainda, que o contato previsto no processo de trabalho do modelo matricial entre as equipes de apoio e as de saúde da família, pelas quais é responsável, é (apenas) de encontros semanais e de formas de contato para demandas inesperadas ou intercorrências (geralmente, via contato telefônico).

Visto a abrangência do número de equipes de saúde da família sob responsabilidade das equipes de matriciamento e o processo de trabalho previsto nesse modelo, identificou-se que se trata de uma atuação característica das especialidades. O tempo e o contato restritos impedem a equipe de apoio matricial de participar do cotidiano das equipes de saúde da família e da comunidade, inviabilizando o desenvolvimento efetivo de um trabalho de promoção e prevenção da saúde. Assim, as equipes de apoio dedicam-se, quase que exclusivamente, a demandas curativas, repetindo o modelo que se pretende superar. As ideias centrais do modelo de apoio matricial (suas intenções) são bastante válidas no sentido de que pontuam a importância de se incluir a saúde mental na atenção básica, e buscam desenvolver um trabalho integrado, promover o diálogo e a articulação entre a atenção básica e os serviços dos níveis secundário e terciário. A questão é que, paradoxalmente, suas diretrizes trazem ações e metas impossíveis de se atingir devido à organização da equipe e ao processo de trabalho que fragmentam a atuação. Com isso, cria-se uma lacuna, distanciando as ações de saúde mental da atenção básica.

A dificuldade de sistematizar um diálogo mais constante entre os serviços propicia o surgimento dos furos na rede de atenção, o que, segundo Marçal (2007), faz com que a percepção do fracasso ou da incapacidade de atender certas demandas seja de responsabilidade de um serviço ou de outro, quando o que ocorre é que determinadas demandas ainda não têm condições de serem resolvidas por estarem nesses furos, por demandarem ações intersetoriais mais estruturadas ou ainda uma co-responsabilização de diferentes serviços, difíceis de serem efetivados, ou mesmo pela falta de investimento financeiro e de trabalhadores para a área.

Uma das consequências diretas desses furos da rede pode ser visualizada nos CAPS, que acabam por centralizar as demandas de saúde mental tornando-se portas de entrada 
sem saída, ou seja, recebem as demandas e encontram dificuldade em referenciá-las para a atenção básica. Nesse contexto, poder-seia pensar nas equipes de apoio em saúde mental como pontes entre as equipes de atenção básica e os serviços de saúde mental. Contudo, entende-se que a organização dessas equipes e seu processo de trabalho reforçam tal dicotomia, na medida em que mantêm a saúde mental como especialidade, e não como parte da saúde integral que deve ser abordada na atenção básica, no cotidiano da unidade local de saúde, na comunidade. No processo de trabalho do apoio matricial em saúde mental, os profissionais da equipe de saúde da família buscam apoio quando surge uma determinada questão em que julguem necessária uma assessoria do especialista em saúde mental, seja para receber orientação, seja para compartilhar o atendimento. Ressalta-se que o foco, nesse processo, é a doença, o sintoma, e a manutenção da saúde mental como especialidade, não como parte da saúde integral. Enquanto se mantiver o foco na doença e nas especializações, não se concretizará a mudança de modelo almejada, pois não se abrirão novas possibilidades de atuação.

Outra dificuldade do matriciamento em saúde mental está na ideia desse modelo de prestar assessoria, supervisionar e instrumentalizar os profissionais da equipe mínima de saúde da família para lidar com as questões de saúde mental. Essa prática corre o risco de tornar-se restrita, tendo em vista os contatos esporádicos entre os profissionais devido ao grande número de equipes a serem atendidas. É possível que médicos e enfermeiros, como quaisquer outros profissionais, sejam instrumentalizados, mas através de trocas cotidianas de um trabalho efetivamente interdisciplinar, pois acredita-se que é no cotidiano de trabalho que se constrói o saber e a prática interdisciplinar que, por sua vez, permite a troca e a instrumentalização mútua entre os profissionais em uma relação horizontal.
Não se pretende negar, em absoluto, os ganhos de muitos Municípios com a implantação do modelo de apoio matricial, sobretudo quando se tem como referência a situação do sistema de saúde anos atrás, em que pouco se falava de saúde mental na atenção básica e quando eram raras as práticas na perspectiva da integralidade e da interdisciplinaridade. $\mathrm{O}$ que se pretende ressaltar são as vantagens da integração efetiva do psicólogo (que tem a interdisciplinaridade e a integralidade como eixos norteadores de sua prática) na atenção básica e as implicações positivas dessa integração para o sistema de saúde.

Argumentos econômicos poderiam ser utilizados para justificar o modelo de apoio matricial, julgando que incluir outros profissionais na equipe mínima de saúde da família representaria um gasto muito grande. Contudo, nesse sentido, o modelo de matriciamento corre o risco de, na prática, ser mais econômico que efetivo, o que, em última análise, deixa inclusive de ser econômico, uma vez que tudo o que se deixa de promover, prevenir e de tratar, na atenção básica, fatalmente sobrecarrega, em algum momento, os demais níveis de atenção. Sendo assim, a inclusão de outros profissionais nas equipes de saúde da família representa um investimento em saúde. Se a atenção básica é o lócus privilegiado de promoção e prevenção, investir em saúde é investir na atenção básica, o que, certamente, prevenirá gastos excessivos e desnecessários nos demais níveis de atenção, que são de alto custo.

Cabe destacar que o Relatório de Gestão (Brasil, 2007b) referente aos avanços e dificuldades, entre 2003 e 2006, do processo de mudança do modelo de atenção à saúde mental revela que os mecanismos de indução financeira, pelo Ministério da Saúde, para o desenvolvimento da política de saúde mental na atenção básica são fundamentais. $\mathrm{E}$ ressalta que esta deverá ser uma das principais prioridades para a expansão e a qualificação 
da saúde mental na atenção básica na gestão 2007 - 2010.

Em entrevista à Revista Brasileira Saúde da Família (2008), ao ser questionado se a atenção primária praticada no SUS apresenta condições para funcionar como centro de comunicação das redes de atenção à saúde, Eugênio Vilaça (consultor em saúde pública) afirma que o ciclo atual da atenção básica, caracterizado pelo desenvolvimento e pela expansão da ESF, tem muitos resultados a comemorar. Contudo, ressalta que esse ciclo se esgotou e não tem condições de estruturar uma prática de atenção primária capaz de desempenhar adequadamente os papéis de um centro de comunicação das redes de atenção à saúde. Dentre os fatores problematizados, aponta o funcionamento com base em cuidados profissionais exclusivamente por médicos e enfermeiras, destacando a ausência de uma equipe interdisciplinar. Segundo o consultor, há que se instituir um novo ciclo na atenção primária no Brasil, o que implicará assumi-la verdadeiramente, e não só discursivamente, como a estratégia de organização do SUS. "A implantação das redes de atenção à saúde no SUS começa por um choque de qualidade na atenção primária à saúde. Isso custará um pouco a mais, em esforços e recursos financeiros, mas agregará valor à população brasileira" (p. 9).

Em outras palavras, a Saúde da Família mostra ser uma estratégia eficiente no caminho da consolidação do SUS, contudo, é preciso avançar, investir, sobretudo em recursos humanos, para qualificar a atenção básica e permitir que esta cumpra seu papel. Frente a essa atribuição, entende-se que a atenção básica necessita contar com equipes de saúde efetivamente interdisciplinares, nas quais o psicólogo tem muito a contribuir (Böing et al., 2009). Para tanto, torna-se imprescindível que o psicólogo esteja locado na unidade de saúde e faça parte da equipe de saúde da família em regime de trabalho em tempo integral.

Cabe pontuar, entretanto, que a locação do profissional de Psicologia na atenção básica como integrante da equipe de saúde da família, por si só, não garante a mudança da lógica de atenção, uma vez que esta se relaciona diretamente a vários fatores apontados na literatura, dentre eles: à formação, cultura e identidade profissional do psicólogo; à capacidade do profissional em flexibilizar e contextualizar seu conhecimento e suas práticas e à sua posição ética e política (Benevides, 2005; Bock, 1993; Calatayud, 1991; Dimenstein, 1998, 2000, 2001, 2003; Franco \& Mota, 2003; Lima, 2005; Moré, 2000; Moré \& Macedo, 2006; Oliveira et al., 2005; Psicologia: Ciência e Profissão, 2006; Romagnoli, 2006). Em suma, a mudança da lógica de atenção relaciona-se diretamente à postura do profissional. Contudo, pretendese ressaltar que a locação do psicólogo na atenção básica através de sua inclusão na equipe de saúde da família poderia viabilizar ao profissional - identificado com a atuação interdisciplinar e integral - a corroboração da lógica da clínica ampliada para efetivar o modelo de atenção da vigilância da saúde.

\section{Considerações finais}

Esta pesquisa buscou contribuir com a ampliação da compreensão da inserção do psicólogo na saúde coletiva através da análise de aspectos históricos e atuais das políticas públicas de saúde. Esse profissional tem um grande potencial de contribuição, contudo, tem sido pouco mencionado nas políticas. A pesquisa permite concluir que a configuração das políticas de saúde não contempla efetivamente o profissional de Psicologia na atenção básica, ou seja, com atuação de acordo com a ESF, exceto nas equipes de atenção básica que atuam nas unidades penitenciárias. O psicólogo é incluído, na quase totalidade dos documentos em que 
é citado, apenas nos níveis secundário e terciário de atenção, sendo que a relação que esse profissional estabelece com a atenção básica, segundo os documentos, se dá através da atribuição de apoio matricial às equipes de saúde da família. Com isso, pode-se presumir que o psicólogo é tido exclusivamente como especialista, e não como um profissional de saúde geral.

O uso do termo especialista, no contexto desta discussão, refere-se à formação acadêmica e às práticas profissionais pautadas no paradigma tradicional da ciência, que refletem uma visão racionalista e determinista através da dicotomia mente-corpo, que busca o conhecimento aprofundado do funcionamento das partes do corpo, gerando intervenções profissionais fragmentadas (a clínica tradicional). Em contrapartida, o termo profissional de saúde geral, ou clínico geral, refere-se ao profissional que, embora tenha uma formação específica que lhe atribui um determinado campo de atuação, busca a compreensão de indivíduos em contextos, através de uma atuação interdisciplinar em que contribui com sua especificidade, refletindo, flexibilizando e contextualizando suas práticas. É essa postura que, na atenção básica, permite o desenvolvimento de uma atenção integral à saúde.

Para o profissional de Psicologia, falta ainda esse entendimento. O desconhecimento do potencial do psicólogo clínico geral se relaciona ao desconhecimento do potencial da Psicologia no campo da promoção e da prevenção da saúde. O entendimento equivocado da saúde mental estritamente como uma especialidade pode ser outro fator que contribui para a dicotomia saúde/saúde mental constatada nas políticas de saúde.

$\mathrm{Na}$ proposta de inclusão do profissional de Psicologia na equipe de saúde da família, deve ficar clara a ideia de psicólogo generalista, que desempenha ações conjuntas e específicas, sobretudo no campo da promoção e da prevenção da saúde e também no da atenção curativa, com foco na família e na comunidade; atua também como mediador no acesso e no acompanhamento dos usuários e familiares nos serviços de níveis secundário e terciário. Nesse contexto, a saúde mental pode ser entendida como campo de atuação do psicólogo não exclusivo a esse profissional, mas apenas um campo de atuação de sua especificidade, em que tem especial contribuição a oferecer e que, com os demais campos multiprofissionais, comporá a abordagem interdisciplinar, através do diálogo, da atuação conjunta no cotidiano de trabalho na unidade local de saúde e na comunidade, possibilitando, assim, a construção de uma atenção à saúde efetivamente integral.

Integrante da equipe de saúde da família, o psicólogo faz a articulação de todos os recursos disponíveis na rede de atenção, mantendo-se como referência do cuidado e organizador da rede. Os CAPS, devidamente territorializados (como estabelecem as diretrizes), recebem a demanda advinda da atenção básica e cumprem a função de apoio matricial naturalmente (discutindo os casos e desenvolvendo um acompanhamento conjunto), à medida que se efetiva o trabalho em rede, o que implica comunicação constante.

A proposta de inclusão do psicólogo na equipe mínima de saúde da família poderia, em uma análise superficial, assumir a conotação de corporativismo e luta de mercado de trabalho, sob o argumento de que outros profissionais já inclusos na equipe poderiam desempenhar as ações de saúde mental - como, de fato, muitas vezes o fazem. Contudo, embora uma consequência direta desta inclusão seja o aumento do mercado de trabalho para os profissionais de Psicologia, há que se compreender, em uma análise mais acurada, que interdisciplinaridade não significa 
ater-se às atividades mais simples - sem especificidade técnica. A interdisciplinaridade envolve ações em comum, mas não nega as especificidades, pelo contrário, é no campo destas que ela se desenvolve. A exploração máxima de cada especificidade é que permite a composição de um trabalho interdisciplinar de qualidade. Assim, a inclusão de outros profissionais na equipe mínima, dentre eles o psicólogo, representa sobretudo o aumento da possibilidade de se compor um trabalho interdisciplinar de qualidade.

Uma atenção integral, como a pretendida pelo SUS, só poderá ser alcançada através da troca de saberes e práticas e de profundas alterações nas estruturas de poder estabelecidas, sendo instituída uma lógica do trabalho interdisciplinar por meio de uma rede interligada de serviços de saúde. A transformação da atenção depende do compromisso dos gestores e, principalmente, da postura dos próprios profissionais em se reconhecerem como atores sociais com potencial de transformar o quadro atual da saúde pública (Bezerra \& Dimenstein, 2008). Com este estudo, pretendeu-se contribuir para a compreensão da atual situação da Psicologia na atenção básica à saúde, possibilitando a construção de argumentos que sirvam de subsídios concretos para profissionais de saúde e gestores nas discussões do papel do psicólogo no primeiro nível de atenção, auxiliando na conquista de condições adequadas a uma atuação condizente com as demandas da população no contexto da atenção básica. Nesse sentido, este estudo representa, também, uma contribuição teórica para a Psicologia, sobretudo nos cursos de graduação, auxiliando na aproximação da formação do psicólogo com as políticas de saúde e no desenvolvimento de uma posição ética e política.

A busca que se faz necessária é a da construção de políticas públicas de saúde mais claras e integradoras no que se refere à Psicologia no setor saúde, definição de papéis do psicólogo a respeito dos três níveis de atenção e sua inclusão nos três níveis e criação de cargos em quantidade suficiente, com salários dignos e equiparados, enfim, uma política efetiva de saúde coletiva. Para isso, é fundamental que os psicólogos se organizem, que seus representantes ajam, para que os responsáveis pelas políticas públicas de saúde, desde a esfera federal até a regional, conheçam as potencialidades da intervenção psicológica na atenção básica e as vantagens da integração de psicólogos nas equipes de saúde da família.

Elisangela Böing*

Mestrado em Psicologia pela Universidade Federal de Santa Catarina, Santa Catarina - SC - Brasil.

Maria Aparecida Crepaldi (Universidade Federal de Santa Catarina)

Pós-Doutorado pelo Hospital das Clínicas de Ribeirão Preto, Universidade de São Paulo, São Paulo - SP - Brasil. E-mail: maria.crepaldi@gmail.com

*Endereço para envio de correspondência:

Departamento de Psicologia. Centro de Filosofia e Ciências Humanas. Universidade Federal de Santa Catarina ,Trindade, Florianópolis - Santa Catarina - SC - Brasil CEP 88010-970.

E-mail: zanziboing@yahoo.com.br

Recebido 24/9/2009, 1a Reformulação 18/1/2010, 2a Reformulação 17/2/2010, Aprovado 28/2/2010. 
Andrade, S. M., Soares, D. A, \& Cordoni Jr., L. (Orgs.). (2001). Bases da saúde coletiva. Londrina, PR: UEL.

Baptista, T. W. de F. (2007). Análise das portarias ministeriais da saúde e reflexões sobre a condução nacional da política de saúde. Cadernos de Saúde Pública, 23(3), 615-626.

Benevides, R. (2005). A psicologia e o Sistema Único de Saúde: quais interfaces? Psicologia \& Sociedade, 17(2), 21-25.

Bezerra, E. N. R., \& Dimenstein, M. (2008). Os CAPS e o trabalho em rede: tecendo o Apoio Matricial na Atenção Básica. Psicologia Ciência e Profissão, 28, 632-645.

Bock, A. M. B. (1993). Eu caçador de mim: pensando a profissão de psicólogo. In M. J. Spink (Org.), O conhecimento no cotidiano: as representações sociais na perspectiva da psicologia social (pp. 280- 291). São Paulo: Brasiliense.

Böing, E. (2009). O psicólogo na atenção básica: uma incursão pelas políticas públicas de saúde brasileiras. Dissertação de Mestrado, Universidade Federal de Santa Catarina, Florianópolis, SC.

Böing, E., Crepaldi, M. A., \& Moré, C. L. O. O. (2009). A epistemologia sistêmica na atuação do psicólogo na atenção básica à saúde. Psicologia: Ciência e Profissão, 29(4), 828-845.

Brasil. Ministério da Saúde. Secretaria Nacional de Assistência Social à Saúde. (1990). ABC do SUS - doutrinas e princípios (Vol. 1/MS). Brasília, DF: Autor.

Brasil. Ministério da Saúde. Secretaria Nacional de Assistência Social à Saúde. Coordenação de Saúde da Comunidade. (1998). Saúde da Família: uma estratégia para reorientação do modelo assistencial. Brasília, DF: Autor.

Brasil. Ministério da Saúde. Secretaria-Executiva. Núcleo Técnico da Política Nacional de Humanização. (2004a). Humaniza SUS: prontuário transdisciplinar e projeto terapêutico. Brasília, DF: Autor. (Série B. Textos Básicos de Saúde)

Brasil. Ministério da Saúde. Secretaria-Executiva. Núcleo Técnico da Política Nacional de Humanização. (2004b). Humaniza SUS: equipe de referência e apoio matricial. Brasília, DF: Autor. (Série B. Textos Básicos de Saúde)
Brasil. Ministério da Saúde. Secretaria de Atenção à Saúde. Departamento de Ações Programáticas Estratégicas. (2004c). Saúde Mental e Atenção Básica: o vínculo e o diálogo necessários. In Saúde mental no SUS: os centros de atenção psicossocial. Brasília, DF: Autor. (Série F. Comunicação e Educação em Saúde)

Brasil. Ministério da Saúde. (2006a). Portaria $n^{\circ} 648$ de 28 de março de 2006. Aprova a Política Nacional de Atenção Básica, estabelecendo a revisão de diretrizes e normas para a organização da atenção básica para o Programa Saúde da Família (PSF) e o Programa Agentes Comunitários de Saúde (PACS). Brasília, DF: Autor.

Brasil. Ministério da Saúde. Secretaria de Atenção à Saúde. Núcleo Técnico da Política Nacional de Humanização. (2006b). Humaniza SUS: documento base para gestores e trabalhadores do SUS (3a ed.). Brasília, DF: Autor. (Série B. Textos Básicos de Saúde)

Brasil. Ministério da Saúde. Secretaria de Atenção à Saúde. Núcleo Técnico da Política Nacional de Humanização. (2007a). Clínica ampliada, equipe de referência e projeto terapêutico singular (2a ed.). Brasília, DF: Autor. (Série B. Textos Básicos de Saúde)

Brasil. Ministério da Saúde. Secretaria de Atenção à Saúde. (2007b). Relatório de Gestão 2003-2006: saúde mental no SUS: acesso ao tratamento e mudança do modelo de atenção. Brasília, DF: Autor.

Brasil. Ministério da Saúde. (2008). Portaria no 154 de 25 de janeiro de 2008. Cria os Núcleos de Apoio à Saúde da Família - NASF. Brasília, DF: Autor.

Calatayud, F. J. M. (1991). La promoción de la salud como problema de la psicologia em la atención primaria. Revista Cubana de Medicina General Integral, 7(4), 362-370.

Coimbra, V. C. C., Oliveira, M. M., Vila, T. C., \& Almeida, M. C. P. (2005). A atenção em saúde mental na estratégia saúde da família. Revista Eletrônica de Enfermagem, 7(1), 113-111. Recuperado em 08 de setembro de 2007, de http://www. revistas.ufg.br/index.php/fen 
Conselho Regional de Psicologia - 12ª Região. (2007). Revista do VI Congresso Nacional da Psicologia (VI CNP): etapa regional de Santa Catarina. Florianópolis: CRP-12.

Dimenstein, M. (1998). O psicólogo nas unidades básicas de saúde: desafios para a formação e atuação profissionais. Estudos de Psicologia, 3(1), 53-82.

Dimenstein, M. (2000). A cultura profissional do psicólogo e o ideário individualista: implicações para a prática no campo da assistência pública à saúde. Estudos de Psicologia, 5(1), 95-122.

Dimenstein M. (2001). O psicólogo e o compromisso social no contexto da saúde coletiva. Psicologia em Estudo, 6(2), 57-63.

Dimenstein, M. (2003). Los (des)caminos de la formación profesional del psicólogo en Brasil para la actuación en la salud publica. Revista Panamericana de Salud Pública, 13(5).

Dimenstein, M., Santos, Y. F., Brito, M., Severo, A. K., \& Morais, C. (nov. de 2005). Demanda em saúde mental em unidades de saúde da família. Mental. (online), 3(5), 2341. Recuperado em 18 setembro, 2007, de http://pepsic. bvs-psi.org.br/scielo.php?script=sci_arttext\&pid $=$ S1679$4272005000200003 \& \operatorname{lng}=$ pt\&nrm $=$ iso

Ferriolli, S. H. T., Marturano, E. M., \& Puntel, L. P. (2007). Family context and child mental health problems in the Family Health Program. Revista de Saúde Pública, 41(2), 251-259.

Franca, A. C. P. de, \& Viana, B. A. (2006). Interface psicologia e programa saúde da família - PSF: reflexões teóricas. Psicologia: Ciência e Profissão. (online), 26(2), 246-257. Recuperado em 18 de setembro de 2007, de http://pepsic. bvs-psi.org.br/scielo.php?script =sci_arttext\&pid $=$ S1414$98932006000200007 \& \operatorname{lng}=$ pt\&nrm $=$ iso

Franco, A., \& Mota, E. (2003). Distribuição e atuação dos psicólogos na rede de unidades públicas de saúde no Brasil. Psicologia: Ciência e Profissão (online), 23(3), 50-59. Recuperado em 17/09/2007, de http://pepsic. bvs-psi.org.br/scielo.php?script=sci_arttext\&pid=S141498932003000300008\&lng=es\&nrm =iso

Gomes, D. C. R. (1997). Equipe de saúde: o desafio da integração. Uberlândia: Ed. da Universidade Federal de Uberlândia.

Lima, M. (2005). Atuação psicológica coletiva: uma trajetória profissional em unidade básica de saúde. Psicologia em Estudo, 10(3), 431-440.

Marçal, C. R. M., Campos, R. O. E., \& Furtado, J. P. (2007). A saúde mental na atenção básica: uma saída para o sofrimento psíquico? Discussão a partir da inserção na assistência e gestão de uma unidade básica de Campinas, SP. Curso de Aprimoramento em Planejamento e Administração em Serviços de Saúde, Universidade Estadual de Campinas, Campinas, SP. Recuperado em 20 de janeiro de 2008, de http://www.fcm.unicamp.br/grupos/saude_mental/artigos/ aprimorandos/1.pdf

Moré, C. L. O. O. (2000). Atendendo à demanda: proposta de um modelo de sistematização de intervenção psicológica junto a postos de saúde comunitária. Tese de Doutorado, Pontifícia
Universidade Católica de São Paulo, São Paulo, SP.

Moré, C. L. O. O., \& Macedo, R. M. S. (2006). A psicologia na comunidade: uma proposta de intervenção. São Paulo: Casa do Psicólogo.

Oliveira, I. F., Dantas, C. M. B., Costa, A. L. F., Gadelha, T. M. S., Ribeiro, E. M. P. C., \& Yamamoto, O. H. (2005). A psicologia, o Sistema Único de Saúde e o Sistema de Informações Ambulatoriais: inovações, propostas e desvirtuamentos. Interação em Psicologia, 9(2), 273-283.

Organização Mundial de Saúde. Organização Pan-Americana de Saúde. (2001). A Resolução de Problemas de Saúde Mental. In Relatório sobre a saúde no mundo 2001 (pp. 79-107). OMS

Pimentel, A. (2001). The method of documental analysis: The use for a historiographycal research. Cadernos de Pesquisa, 114, 179-195.

Psicologia: Ciência e Profissão. Diálogos. (2006, dezembro). 3(4). (Conselho Federal de Psicologia)

Revista Brasileira Saúde da Família. (2008, julho/setembro). 9(19). (Ministério da Saúde)

Romagnoli, R. C. (2006). A formação dos psicólogos e a saúde pública. Pesquisas e Práticas Psicossociais, 1(2). Recuperado em 08 de setembro de 2007, de http://www.ufsj.edu.br/ Pagina/ppp-lapip/Arquivos/RobertaRomagnoli.pdf

Teixeira, J. A. C. (2000a). Estágios de Psicologia em Centros de Saúde. In I. Trindade \& J. A. C. Teixeira, Psicologia nos cuidados de saúde primários (pp. 103-106). Lisboa: CLIMEPSI.

Teixeira, J. A. C. (2000b). Abordagem psicológica do doente psiquiátrico em centros de saúde. In I. Trindade \& J. A. C. Teixeira, Psicologia nos cuidados de saúde primários (pp. 55-66). Lisboa: CLIMEPSI.

Trindade, I. (2000). Competência do psicólogo nos cuidados de saúde primários. In I. Trindade \& J. A. C. Teixeira, Psicologia nos cuidados de saúde primários (pp. 37-46). Lisboa: CLIMEPSI.

Trindade, I., \& Teixeira, J. A. C (2000a). Aconselhamento psicológico nos cuidados de saúde primários. In I. Trindade \& J. A. C. Teixeira, Psicologia nos cuidados de saúde primários (pp. 67-80). Lisboa: CLIMEPSI.

Trindade, I., \& Teixeira, J. A. C. (2000b). Intervenção psicológica em centros de saúde. In I. Trindade \& J. A. C. Teixeira, Psicologia nos cuidados de saúde primários (pp. 23-36). Lisboa: CLIMEPSI.

Universidade Federal de Santa Catarina. (2007). Programa de Residência Integrada em Saúde da Família. Centro de Ciências da Saúde. Florianópolis. (Mimeo)

Valles, M. S. (1997). La investigación documental: Técnicas de lectura y documentación. In M. S. Valles, Técnicas cualitativas de investigación social: reflexión metodológica y práctica profesional (pp. 109-141). Madrid: Editorial Síntesis. 